\title{
7 Schlussbemerkung
}

Der Schriftsteller Curt Riess, ein Freund der Ullsteins, hatte seine eigene Theorie, ab welchem Punkt die Probleme mit der Ullstein-Restitution aus dem Weg geräumt waren. Das war, nach seiner Erinnerung, zur Jahresmitte 1951. Und er persönlich hatte dafür gesorgt:

Rund eine Woche später, wieder nach Berlin zurückgekehrt, gab ich eine kleine Gesellschaft. Ich lud ein: Rudolf Ullstein und seine Frau, Karl Ullstein und seine Frau, Heinz Ullstein, die bekannte Schauspielerin Heidemarie Hatheyer und - Ernst Reuter und seine Frau. Die Villa, in der dieses Abendessen stattfand, war nicht gerade sehr geräumig, man hatte sozusagen Tuchfühlung. Reuter unterhielt sich mit Karl Ullstein - Rudolf war früher gegangen - bis gegen zwei Uhr morgens. [...] Was im Einzelnen besprochen wurde, wissen wohl nur die beiden - und heute weiß es wohl niemand mehr. Aber von diesem Augenblick an änderte sich die Atmosphäre. Es durfte als sicher gelten, dass Reuter den Ullsteins keinerlei Schwierigkeiten mehr bereiten würde. ${ }^{1}$

Diese romantisierende Verklärung der Ereignisse steht stellvertretend für all die seichten Anekdoten und Legenden, die im Laufe der Zeit rund um die Restitution des Ullstein-Verlags entstanden sind. Doch die wahren Umstände der Rückübertragung waren kompliziert und verstörend, geprägt von Wirtschaftsinteressen, politischem Kalkül und mangelndem Schuldbewusstsein.

Welche Erkenntnisse lassen sich also im Einzelnen aus der Studie ziehen?

Die Bestimmungen der von den Alliierten erlassenen Rückerstattungsanordnung (REAO) aus dem Juli 1949 hatten mannigfaltigen Einfluss auf den Verlauf und das Ergebnis des Restitutionsvergleichs. Zwei Artikel stechen dabei besonders hervor: Art. 3 und Art. 30 REAO.

Die im Art. 3, Abs. 2 und Abs. 3 REAO dargelegten Ausnahmen von einer „ungerechtfertigten Entziehung“ hatten den Gegenparteien der Ullstein-Restitution vermeintliche Gründe gegen eine Wiedergutmachung geliefert. Die Berliner Landesregierung kooperierte dafür mit Max Winkler, dem ehemaligen Geschäftsführer der Ullstein-Erwerberin, der Cautio GmbH. Dessen Aussage am Rande des Wilhelmstraßen-Prozesses, wonach er Franz Ullstein geholfen habe, Teile des Kaufpreises ins Ausland zu transferieren, schürte innerhalb der Familie bewusst die Vorbehalte gegen den Stamm III - die offene Fehde zwischen den Brüdern war schließlich seit den Aufsehen erregenden Gerichtsprozessen der Jahre 1930/31 kein Geheimnis. Und tatsächlich beschreiben zahlreiche hausinterne Vermerke und Protokolle, etwa anlässlich der Generalversammlung vom Juli 1950, die Folgen der Verschärfung dieser Auseinandersetzung. Das ge-

1 Nach Riess, Restitution, S. 411.

๖ Open Access. () 2020 Juilane Berndt, publiziert von De Gruyter. (๕) By Dieses Werk ist lizenziert unter der Creative Commons Attribution-NonCommercial-NoDerivatives 4.0 License. 
meinsame Agieren der fünf Stämme bei den Alliierten - eine unentbehrliche Voraussetzung für die Restitution - wurde dadurch massiv behindert.

Es war nur konsequent, dass der Berliner Senat die Vergleichsverhandlungen dazu benutzte, um eine Entschädigungszahlung an Winkler über 10.000 DM zu erwirken. So mussten die Ullsteins, deren schwierige Liquidität hier ausführlich umrissen wurde, den Mann, der 1934 den Zwangsverkauf ihres Imperiums eingefädelt hatte, auch noch ausbezahlen.

Die Auswirkung der anderen für die Ullstein-Restitution besonders relevanten Bestimmung, des Art. 30 REAO, waren ebenfalls gravierend. Die darin aufgeführte Belastungsgrenze hätte eigentlich einen gesunden finanziellen Neustart des Unternehmens nach der Restitution ermöglichen sollen, doch die Ullsteins sahen sich verpflichtet, die in der Phase der Treuhänderschaft entstandenen Schulden zu übernehmen. Selbst für die von den nationalsozialistischen Besatzern in Frankreich geraubte Wifag-Druckmaschine sahen sich die Ullsteins in der Verantwortung - kein Wunder, war sie doch zum Zeitpunkt der Restitution eine der wichtigsten Vorrichtungen im Druckhaus Tempelhof.

Eine konkrete Anwendung des Art. 30 REAO hätte beinhaltet, dass auch die Verbindlichkeiten gegenüber den Angestellten und Arbeitern des Deutschen Verlags für nichtig erklärt worden wären. Doch das kam für die Ullsteins nicht infrage - man hatte schließlich einen Ruf zu verlieren.

Der Senat war sich dessen bewusst - und kalkulierte mit ein, dass die Ullsteins die Schulden des Deutschen Verlags bei der öffentlichen Hand in siebenstelliger Höhe unter Missachtung der Bestimmungen des Art. 30 REAO übernehmen würden. Erneut sei hier die senatsinterne Notiz über die Große Anfrage im Berliner Abgeordnetenhaus im März 1952 zitiert:

Im Falle Ullstein war die Belastungsgrenze [...] weit niedriger als die Summe der Verpflichtungen des Unternehmens Deutscher Verlag, das Ullstein zurückerhielt. Dem Senat ist es im Vergleichswege gelungen zu erreichen, dass die Familie Ullstein trotz des Rechts auf ihrer Seite Verpflichtungen in Höhe eines Mehrfachen der Belastungsgrenze übernahm. Ullstein lag dabei an einer raschen Restitutionsentscheidung. Wäre es zur Durchführung des Rechtsverfahrens vor den Wiedergutmachungsinstanzen gekommen [und nicht zum Vergleich der Parteien, d. Verf.], so hätte nach dem Gesetz entschieden werden müssen und der Senat von Berlin wäre unzweifelhaft viel schlechter gefahren. ${ }^{2}$

Die amerikanischen Besatzungsbehörden übten einen störenden Einfluss auf die Restitutionsbestrebungen der Ullsteins aus. Dafür fand diese Studie zahlreiche Beispiele. Die Alliierten benötigten das Druckhaus Tempelhof für ihre Reeducation-Politik - und wendeten somit bereits in der unmittelbaren Nachkriegs-

2 Vgl. undatierte Notiz zur Großen Anfrage im Abgeordnetenhaus über den Grundstückskauf, Verfasser ungenannt, wahrscheinlich aber Hans Hirschfeld, in: LAB B Rep. 002 Nr. 4102. 
zeit präventiv jeden möglichen Anspruch ab, den die Ullsteins auf dieses Herzstück ihres alten Verlages hätten erheben können. Die Druckerei war vor allem für die Amerikaner ein wichtiges Instrument, um die von ihnen lizenzierten Neu-Verleger mit günstigen Druckverträgen zu subventionieren. Damit die Besitzansprüche der Familie Ullstein erst gar nicht belastbar untermauert werden konnten, enthielt man folglich ihnen und den Treuhändern des Deutschen Verlags die betriebswirtschaftlich wichtigen Tageszeitungslizenzen vor. Nicht einmal Heinz Ullstein, ein anerkanntes Opfer des NS-Regimes, bekam die Chance auf die Herstellung einer Tageszeitung.

Wie dargelegt, verweigerte man Rudolf Ullstein mehrfach die Zuzugsgenehmigung für Berlin - obwohl zu diesem Zeitpunkt die Rückerstattungsanordnung bereits in Kraft war. Und die Maßnahmen der Amerikaner im Zuge der Blockade ließen durchaus $z u$, von einer Zwangsverstaatlichung des Unternehmens zu sprechen, schließlich wurden die Einrichtungen und das Personal des Deutschen Verlags in dieser Zeit unentgeltlich genutzt, um Energie in das Berliner Elektrizitätsnetz einzuspeisen. Dass man zudem dafür sorgte, dass die Herausgeber des Tagesspiegels den Großteil ihrer während der Blockade aufgelaufenen Schulden nicht bezahlen mussten, während sich die Verschuldung des Deutschen Verlags bei der öffentlichen Hand existenzbedrohend erhöhte, unterstreicht die Präferenzen der US-Stadtkommandantur: Hier ging es nicht um Gerechtigkeit, sondern um den Schutz der durch die Amerikaner lizenzierten NeuVerleger.

Den traurigen Höhepunkt der Kampagne zugunsten der Neu-Verleger stellte die Verhinderung der Weitergabe des Restitutionsantrags an die Wiedergutmachungsämter durch Joseph McNulty, den Leiter von Property Control, im März 1950 dar. $^{3}$ Wie geschildert, zwang man den Antragstellern zunächst einen Schuldenschnitt über 400.000 DM für den Tagesspiegel auf, bevor man ihren Antrag endlich an die zuständigen deutschen Behörden weiterleitete. Zudem musste der Deutsche Verlag die Druckpreise für das Vorzeigeblatt des publizistischen Neubeginns in Berlin um $13 \%$ verringern. ${ }^{4}$

Erst die massive Überschuldung des Deutschen Verlags veränderte die Haltung der US-Behörden gegenüber einer Restitution der Ullstein AG - und beendete deren fragwürdiges Vorgehen. Das Zustandekommen der Wiedergutmachung war somit letzten Endes das Ergebnis wirtschaftlicher Notwendigkeiten und eben keine Entscheidung, die aus juristischen oder moralischen Erwägungen heraus getroffen wurde.

3 Schreiben per Boten von Ullmann an Ruge vom 30.03.1950, in: AS-UA, Bestand Ullstein, Band 16.

4 Aktennotiz von Heinz Ullmann vom 13.04.1950, in: AS-UA, Bestand Ullstein, Band 16. 
Das direkte Umfeld des Hohen Kommissars John McCloy beeinflusste den Restitutionsvorgang maßgeblich und direkt. Obwohl die juristischen Parameter abgesteckt und die zuständigen Instanzen sich des Falls offiziell angenommen hatten, unterstreicht der Verlauf der Ullstein-Restitution, in welchem Umfang sich die oberste Führung der Militärregierung dennoch in einen Wiedergutmachungsfall einzumischen vermochte.

Zwei widerstreitende Pole aus dem direkten Umfeld von US-Hochkommissar John McCloy übten massiven Einfluss aus. Shepard Stone, sein Sonderberater für öffentliche Angelegenheiten und Informationswesen, nahm nach Rücksprachen mit Hans Hirschfeld, dem Leiter des Senatspresseamts, eine hemmende Funktion ein: Er zog die bereits gewährte Mehrheitsbeteiligung des Deutschen Verlags am Berliner Anzeiger ohne Begründung zurück, unterstützte die Bildung des „Sonderausschusses Ullstein“, um einen Verkauf des Druckhauses Tempelhof an die Neu-Verleger einzufädeln, und offerierte seine Einflussnahme nach der Wiedereinführung der Berliner Morgenpost, die einen Zeitungskrieg ausgelöst hatte.

Stones Gegenpol war der Jurist Fritz Ernst Oppenheimer. Erst seine direkte Einflussnahme bei seinem alten Weggefährten McCloy - eine durch die Ullstein-Familie bezahlte PR-Maßnahme - beendete Stones Aktivitäten. Praktisch über Nacht war der Reuter-Senat im Oktober 1951 endlich bereit und willig, einen Vergleich mit den Ullsteins zu erreichen.

Der Berliner Senat und seine Verwaltung standen der Ullstein-Restitution ablehnend gegenüber. Die Landesregierung verbündete sich mit den ihr politisch nahestehenden Neu-Verlegern, um das Wiederaufleben der Ullstein AG aktiv zu behindern. Die Berliner Behörden stellten die Umstände des Zwangsverkaufs von 1934 öffentlich in Frage und eigneten sich damit die Argumentation des nationalsozialistischen Regimes an. Mit Hans Hirschfeld hatten die Ullstein-Konkurrenten zudem einen einflussreichen Mitstreiter in ihren Reihen, der die Ressourcen des Senats und seine persönlichen Beziehungen zu Angehörigen der Militärregierung dazu nutzte, um die Restitution aktiv zu behindern. Folgerichtig bildete er mit den Neu-Verlegern, die größtenteils der SPD nahestanden, und den Amerikanern einen Sonderausschuss als effektives Bündnis gegen die Ullsteins.

Im politisch höchst angespannten Klima des ausbrechenden Kalten Krieges wusste der Taktiker Hirschfeld ganz genau, welche Auslöser er betätigen musste, um die Ullsteins bei der Militärregierung zu diskreditieren. Als er in seinen Briefen an seinen Freund Shepard Stone, der auch nach dem Ausscheiden aus dem aktiven Militärdienst großen Einfluss bei den Besatzungsbehörden besaß, vor Weimarer Verhältnissen in West-Berlin warnte und über das „Ruhrkapital“ raunte, das vermutlich hinter den Ullsteins stünde, wusste Hirschfeld ganz ge- 
nau, was er damit bewirkte. Man beraubte die Familie mittels der Konstruktion außenpolitischer Vorwände ihres finanziellen Spielraums und vereinnahmte das amerikanische Hochkommissariat gegen die Ullsteins.

Für diese Studie war es ein Glücksfall, im Berliner Landesarchiv auf eindeutige Dokumente zu stoßen, die keine Zweifel an der Intention und Vorgehensweise des Senats lassen. Den nachhaltigsten Eindruck hinterließ das Schreiben Hans Hirschfelds an Tagesspiegel-Herausgeber Franz Karl Maier vom 27. Oktober 1951, in dem er eingestand, dass...

...der Regierende Bürgermeister mir gegenüber den Standpunkt noch einmal bekräftigt [hat], dass wir heute den vorgeschlagenen Vergleich gar nicht mehr länger hinausziehen können. In dem Augenblick, wo die politischen, Ihnen ja bekannten Bedenken wegfallen durch bindende Erklärungen des Herrn K. U. [= Karl Ullstein, d. Verf.], müssen wir die Sache ihren Weg gehen lassen und können uns nicht hinter neuen Ausflüchten verstecken. Das verträgt weder das politische Ansehen des Regierenden Bürgermeisters, noch würde es im Einklang mit seinen wiederholten festen Zusicherungen gegenüber Herrn K. U. wie Herrn Ullmann stehen. [...] Es ist nichts bis jetzt geschehen, aber wir könnten, wenn der Finanzsenator heute den Vergleich im Senat vorbringt, nichts mehr dagegen einwenden. Sie verstehen sicher diesen Standpunkt und werden begreifen, dass von hier aus nichts geschehen wird, um die Sache zu beschleunigen, es kann aber auch kaum mehr etwas geschehen, um sie zu verlangsamen. ${ }^{5}$

Die Berliner Landesregierung agierte nicht nur parteiisch - sie gestand hier ein, die Ullstein-Restitution aktiv behindert und hinausgezögert $\mathrm{zu}$ haben.

Die verzögerte Restitution samt ihrer finanziellen Folgen belastete das wiedererstandene Unternehmen massiv. Natürlich ließe sich spekulieren, dass die Ullsteins selbst bei einer schnellen Restitution und einer Finanzierung durch französische Investoren auf dem umkämpften West-Berliner Zeitungsmarkt, dem das Umland verloren gegangen war, ohnehin zum Scheitern verurteilt gewesen wären.

Tatsache ist jedoch, dass man den Ullsteins mit Vorsatz alle erdenklichen Steine auf dem Weg zur Restitution in den Weg legte. Dass man ihnen die während der Blockade aufgelaufenen Schulden des Deutschen Verlags überhalf und die Immobilien im ehemaligen Stadtzentrum als Kompensation einbehielt. Dass man ihnen die Möglichkeit verbaute, Investitionspartner im Ausland zu suchen.

Aufgrund dieser mannigfaltig belasteten Ausgangssituation erscheint das wirtschaftliche Versagen der wiedererstandenen Ullstein AG in den 1950er Jahren in einem anderen Licht. Das Unrecht, das den Ullsteins unter dem nationalsozialistischen Regime widerfahren war, wirkte nach dem Untergang fort - und

5 Brief Hirschfelds an Franz Karl Maier vom 27.10.1951, in: LAB B Rep. 002 Nr. 4102. 
erhielt durch die Geschehnisse vor, während und nach der Restitution neue und verstörende Facetten.

Die Rolle der Neu-Verleger in der unmittelbaren Nachkriegszeit war im vorliegenden Fall zwiespältig und bedarf grundsätzlich einer differenzierteren Betrachtung. Die stärkste Kraft gegen die Restitution bildeten zweifelsohne die Neu-Verleger. Sie streuten in ihren Zeitungen Gerüchte über „geheime Finanzquellen“ der Ullsteins, sie diskreditierten die Familie im Lizenzierungsausschuss, indem man ihr die demokratische Gesinnung absprach. Die Konkurrenten hielten den Ullsteins, die in der Emigration neue Staatsbürgerschaften angenommen hatten, vor, keine Deutschen und damit pauschal nicht vertrauenswürdig zu sein.

Die Neu-Verleger waren dabei nicht vor der Verwendung von Feindbildern und Klischees gefeit, welche antisemitischen Stereotypen nahekommen. Dies wurde etwa in der Titelseiten-Veröffentlichung und zustimmenden Kommentierung der Pressemitteilung Fritz Heines im Dezember 1952 im Telegraf deutlich: Demnach sei die „einzige Aufgabe“ Karl Ullsteins...

„...darin zu erblicken, den demokratischen Zeitungen West-Berlins durch sein rücksichtloses und vom reinsten Geschäftsegoismus diktierte Vorgehen die materielle Grundlage zu zerstören. [...] Jeder Versuch aber, mit Hilfe unkontrollierbarer Millionengelder ein Zeitungsmonopol zu errichten, müsse den schärfsten Widerstand aller hervorrufen, denen die demokratische Pressefreiheit und deren Sicherung unantastbar seien. [...] Der Verlag Ullstein vertritt den Standpunkt: Alle Mittel gelten. [...] Gebraucht werden aber nur die Ellenbogen, um die beiseite zu stoßen, die sich nicht 1952, sondern bereits 1946 bewährten $[. .$.$] . “6$

Der „Geschäftsegoismus“ und die vermeintliche Ellenbogenmentalität derjenigen, die sich erfrechten, ihr angestammtes Recht einzufordern, sowie das Raunen über geheime Finanzquellen bedienen antisemitische Stereotypen. Dieses angeblich ich-bezogene Agieren der Ullsteins für eine Rückkehr in ihr angestammtes Metier wird hier in scharfem Widerspruch zur Idee des demokratischen Neubeginns in Berlin gesetzt.

Denn während die Neu-Verleger die Fahne der Freiheit hochhielten, spielt die verlegerische Tätigkeit der Ullsteins nur den Russen und damit den Feinden der Demokratie in die Hände: „Die demokratischen Zeitungen Berlins erfüllten und erfüllen in den schwersten Stunden dieser Stadt ihre Mission, die Stimme der Freiheit zu sein. Wer an ihrem Bestand rüttelt, leistet den Sowjets Schützenhilfe. Was aber bisher den Kommunisten nicht gelungen sei, äußerte Heine, dürfte Herrn Ullstein nicht gestattet werden. ${ }^{\text {“7 }}$

6 Siehe „Provozierend und Gefährlich - Fritz Heine über die Praktiken des Ullstein-Verlages“, in: Der Telegraf, Ausgabe vom 17.12.1952, S. 1. 
Die einzigen jedoch, denen man hier mit Fug und Recht wirtschaftliche Motive gepaart mit intransparenten Finanzquellen unterstellen könnte, wären die Neu-Verleger selbst, die vergeblich versucht hatten, mithilfe der Amerikaner das Druckhaus Tempelhof zu erwerben. Berücksichtigt man dann noch die hier erlangten Erkenntnisse über die unfairen Bevorzugungspraktiken zugunsten der Neu-Verleger - wie die verhinderte Weitergabe des Restitutionsantrags, um dem Deutschen Verlag einen Schuldenschnitt für den Tagesspiegel abzupressen, oder die zwangssubventionierten Druckkosten für die Lizenzzeitungen -, deutet dies die Notwendigkeit einer differenzierteren Beurteilung der publizistischen Gesamtsituation in der unmittelbaren Nachkriegszeit an.

Diese Studie mag somit als ein Plädoyer dafür dienen, die Rolle und das Agieren der Neu-Verleger generell kritischer zu betrachten - über die rein biografischen Aspekte ihrer vermeintlichen Unbelastetheit hinaus.

Erst seit der Jahrtausendwende ist es zu einer dezidierteren Erforschung der Remigration jüdischer Emigranten und Emigrantinnen in den deutschsprachigen Raum gekommen. ${ }^{8}$ Von besonderer Bedeutung war dabei die Rolle der Rückkehrer für den Aufbau demokratischer Strukturen und moderner Einstellungsmuster.

Der Restitutionsfall Ullstein nun bestätigt wesentliche Erkenntnisse der wissenschaftlichen Aufarbeitung der jüdischen Remigration nach dem Zweiten Weltkrieg. Das hier geschilderte widerstrebende Verhalten der Berliner Behörden reflektierte die damals unter Beamten weit verbreitete Haltung, wonach diese „den Unrechtscharakter ihres einstigen Vorgehens“ schlichtweg nicht wahrhaben wollten und sich darum gegenüber jüdischen Antragstellern schikanös verhielten. ${ }^{9}$ Rund 150.000 Beamte, die zuvor in nationalsozialistischen Diensten gestanden hatten, nie rechtskräftig verurteilt oder in eine der ersten beiden Entnazifizierungskategorien eingeordnet worden waren, durften aufgrund des 1951 erlassenen „Gesetzes zur Regelung des Rechtsverhältnisses der unter Artikel 131 des Grundgesetzes fallenden Personen“ in den öffentlichen Dienst zurückkehren.

7 „Provozierend und Gefährlich - Fritz Heine über die Praktiken des Ullstein-Verlages“, in: Der Telegraf, Ausgabe vom 17.12.1952, S. 1.

8 Unter anderem Krauss, Marita: Heimkehr in ein fremdes Land. Geschichte der Remigration nach 1945. München 2001; Biller, Marita: Remigranten in der Publizistik im Nachkriegsdeutschland. In: Rückkehr und Aufbau nach 1945.Deutsche Remigranten im öffentlichen Leben Nachkriegsdeutschlands. Hrsg. von Claus-Dieter Krohn u. Patrik von zur Mühlen. Marburg 1997, S. $275-287$.

9 Herzig, Arno: Jüdische Geschichte in Deutschland. Von den Anfängen bis zur Gegenwart. München 1997, S. 265. 
In der Zeit des Nationalsozialismus waren rund 500.000 Menschen aus dem deutschsprachen Teil Europas emigriert, nur etwa $5 \%$ davon kehrten zurück..$^{10}$ Wie hier geschildert, hatte die Mehrzahl der im Exil lebenden Ullsteins bereits während des Krieges versucht, ihre Rückkehr nach Deutschland vorzubereiten. Doch wie so viele andere Emigranten bekamen sie das Misstrauen und die Vorbehalte der Verbliebenen zu spüren; wie viele andere Rückkehrer mussten sie zur Kenntnis nehmen, dass der Antisemitismus unter den Deutschen ungebrochen war. Sie wurden mit Schuld und Verdrängung konfrontiert, nicht zuletzt spiegelte sich dies in der Pressekampagne gegen das Wiederaufleben des Ullstein-Verlags oder in der zögerlichen Lizenzerteilung wider: Die Rückkehrer, so der Vorwurf, hätten Deutschland in dessen dunkelster Zeit verlassen und somit den einfacheren, ja bequemeren Weg gewählt. ${ }^{11}$

Alles in allem lässt sich subsummieren, dass der Fall Ullstein wahrhaftig kein Ruhmesblatt für die Wiedergutmachungspolitik darstellt. Er reflektiert vielmehr die bittere Realität der Nachkriegsjahre - und das Versagen der Institutionen, sich ihrer moralischen Verantwortung zu stellen.

Zum Abschluss der Studie bleibt noch festzuhalten, was heute vom Ullstein-Verlag, diesem Medien-Giganten der wilhelminischen Ära und der Weimarer Republik, übrig ist. Die Zeiten, in denen veritable Zeitungskriege und politische Auseinandersetzungen um Tageszeitungen und ihre Auflagen geführt wurden, scheinen heute, nur zwei Generationen später, im Zeitalter der Digitalisierung und des Zeitungssterbens, genauso weit entfernt wie die Aufregung um den Prager Fenstersturz oder die Emser Depesche. Der Medienwandel des frühen 21. Jahrhunderts spiegelt sich im Schicksal der Ullstein-Publikationen wider.

Die Buchverlage der Ullstein-Gruppe, zu denen damals u.a. die Marken Heyne, Propyläen, Ullstein, Claassen, List, Econ sowie Marion von Schröder gehörten, wurden 2003 an Bertelsmann, respektive an den Medienkonzern Bonnier veräußert. Der Großteil der Gruppe firmiert heute unter dem Dach des Letztgenannten unter dem Namen Ullstein Buchverlage GmbH. Einige Marken verschwanden, neue, wie der Selbsthilfe-Verlag Allegria, kamen hinzu. Seit dem Jahr 2014 erweitern die Ullstein Buchverlage ihr Portfolio zudem um digi-

10 Von der Lühe, Irmela, Schildt, Axel u. Stefanie Schüler-Springorum (Hrsg.): „Auch in Deutschland waren wir nicht wirklich zu Hause“. Jüdische Remigration nach 1945. Göttingen 2008 (Hamburger Beiträge zur Geschichte der deutschen Juden 34), S. 9.

11 Siehe Bergmann, Werner: „Wir haben sie nicht gerufen“. Reaktionen auf jüdische Remigranten in der Bevölkerung und Öffentlichkeit der frühen Bundesrepublik. In: „Auch in Deutschland waren wir nicht wirklich zu Hause“. Jüdische Remigration nach 1945. Hrsg. von Irmela von der Lühe, Axel Schildt u. Stefanie Schüler-Springorum. Göttingen 2008 (Hamburger Beiträge zur Geschichte der deutschen Juden 34), S. 19-40, hier S. 21. 
tale Imprints. Auch wenn sich der Name Ullstein also nach mehr als 300 Jahren langsam vom gedruckten Wort und vom Papier zu verabschieden beginnt, genießt der Berliner Verlag im Buchhandel wie eh und je den besten Ruf: Mit ihren Krimis dominieren Ullstein-Autoren wie John le Carré, Nele Neuhaus oder Jo Nesbo auch heute noch die Bestsellerlisten.

Unberührt von dem Verkauf im Jahr 2003 war die wichtige Ullstein-Zeitungssparte, die bei Axel Springer verblieb und zu der, wie in den 1950er Jahren, die Berliner Morgenpost und die B.Z. gehörten. Doch die Auflagenerosion bei den Tageszeitungen führte auch hier zu massiven Veränderungen: Im Mai 2014 veräußerte die Axel Springer SE die Berliner Morgenpost zusammen mit dem Hamburger Abendblatt an die Funke Mediengruppe. Bereits ein Jahr zuvor, im Juli 2013, gab man bekannt, dass die Redaktion der B.Z. mit der der BILDRegionalausgabe Berlin zusammengelegt werde. In der entsprechenden Pressemitteilung betonte man: „Beide Titel behalten ihre publizistische Eigenständigkeit. "12 So bleibt die B.Z., wenn auch nicht mehr mit eigener Redaktion, zumindest als Marke erhalten - sie ist der letzte verbliebene Ullstein-Titel bei Axel Springer.

Gut möglich, dass das - trotz der massiven Digitalisierungsstrategie, die die Axel Springer SE heute betreibt - auch künftig so bleibt. Dafür spricht zumindest ein berühmtes Gemälde, das im Journalisten-Club in der 19. Etage des Firmensitzes in Berlin-Kreuzberg hängt.

Das Bild zeigt Leopold Ullstein, honorig schwarz gekleidet vor dunkelbraunem Hintergrund, mit strengem und etwas melancholischem Blick. Gemalt hatte es Oscar Begas 1882, ein Jahr, nachdem Ullstein mit seinem expandierenden Verlag in die Kochstraße 23 gezogen war. Auf der Rückseite des Gemäldes ist ein Brief befestigt. ${ }^{13}$ Die Familie Ullstein hat darin verfügt, dass das Porträt als Dauerleihgabe im Axel Springer Verlag verbleiben darf, solange man hier den Namen Ullstein in Ehren halte.

12 Siehe Pressemitteilung „Digitale Offensive: B.Z. und Berlin-Redaktion von BILD bündeln ihre Kräfte“ der Axel Springer SE vom 24.07.2013. https://www.presseportal.de/pm/6338/ 2520778 (18.07.2018).

13 Vgl. Berndt, Axel Springer, S. 95. 
